\title{
New cell or new cycle?
}

\author{
Olivier Ganier and Marcel Mechali ${ }^{1}$
}

Institute of Human Genetics, CNRS, 34396 Montpellier, France

In mammals, trophoblast giant (TG) cell differentiation is characterized by a physiological endoreduplication, resulting in genome size augmentation. A recent study by Ullah and colleagues (pp. 3024-3036), published in this issue of Genes \& Development, now elucidates the role of the cyclin-dependent kinase inhibitors (CKIs), p21 and p57, in mammalian endocycle regulation.

An important rule during cell division is to replicate DNA once and only once. A variety of robust mechanisms exist to ensure that cells correctly discriminate between replicated and not yet replicated sequences (DePamphilis et al. 2006). Initiation of DNA replication requires the assembly of prereplication complexes (preRCs) at replication origins during the M-to-G1 transition, when global Cyclin-dependent kinase (CDK) activity and expression of the pre-RC formation inhibitor, Geminin, are low. Then, S-phase entry is stimulated by Dbf4-dependent kinase (DDK) and CDK activities, and origin firing is triggered. During S-phase progression, reloading of preRCs is hindered, both by binding of Geminin as well as the inhibition of multiple crucial pre-RCs components like CDT1, CDC6, and ORC1. The introduction of a replication stress, such as DNA damage, induces the ATR-CHK1 pathway to inhibit the CDC25 phosphatase and CDK1, thereby delaying mitosis until the $S$ phase is correctly completed. Failure to comply with this rule induces genome instability and uncontrolled gene amplification, which potentially can lead to cancer (Calvi 2006).

However, what is absolutely forbidden during the mitotic cell cycle is sometimes both useful and necessary during development or differentiation. Hence, endoreplication, the mechanism by which cells exit from the mitotic cell cycle and start to replicate their DNA without cell division, may be an integral part of a normal differentiation program (for review, see Edgar and OrrWeaver 2001). Endocycles are characterized by successive rounds of DNA replication, separated by a Gap phase, ultimately leading to polyploid cells. Endocycles are observed during development of several animal species, from Drosophila to humans, as well as in plants, although

[Keywords: Endoreduplication; placentomegaly; trophoblast giant cell; p57; CDK1; DNA replication]

Corresponding author.

E-MAIL mechali@igh.cnrs.fr; FAX 33-4-99-61-99-20.

Article is online at http://www.genesdev.org/cgi/doi/10.1101/gad.1740808. their purpose in plant development and the mechanism used to regulate endoreplication are not conserved between plants and animals.

In mammals, only few cell types become polyploid. Among them, only megakaryocytes and trophoblast giant (TG) cells can endoreplicate their DNA to reach ploidies with a DNA content $>1000$-fold higher than that of a normal diploid cell (Edgar and Orr-Weaver 2001). During placenta formation, trophoblast stem (TS) cells, derived from the blastocyst, undergo endoreplication in their differentiation into TG cells. This process is regulated by FGF4 deprivation and can be recapitulated in vitro (Fig. 1; Simmons and Cross 2005). In this issue, DePamphilis's group (Ullah et al. 2008) reports that specific inhibition of CDK1 is sufficient to induce TS cells to endoreduplicate and differentiate into TG cells. This effect appears to be cell-specific, as inhibition of CDK1 in other cell types does not lead systematically to polyploidy but rather to cell cycle arrest at the G2-M stage and apoptosis (Goga et al. 2007; this study).

\section{Rereplication in metazoan cells by deregulation of origin firing}

Polyploidization in metazoan cells can result from alterations at various points during the cell cycle. First, rereplication may occur by illegitimate reinitiation of DNA replication at DNA replication origins within the same cell cycle. Here, the targets are the pre-RCs that assemble at replication origins during the G1 period of the cell cycle. CDT1 and Geminin are key regulators of this assembly. Degradation of Geminin at mitosis allows reactivation of the licensing process by CDT1. Therefore, the CDT1/Geminin balance must be accurately controlled during cell cycle progression, as overexpression of CDT1 or inhibition of Geminin triggers the refiring of DNA replication origins during $S$ phase in different metazoan cell types (e.g., Drosophila [Mihaylov et al. 2002; Thomer et al. 2004], Xenopus [Arias and Walter 2005; Li and Blow 2005; Maiorano et al. 2005], and human cells [Zhu et al. 2004]). Mouse embryos, in which Geminin has been genetically disrupted, also spontaneously rereplicate and activate a DNA damage checkpoint mechanism that induces apoptosis (Gonzalez et al. 2006; Hara et al. 2006). However, in human cells, rereplication induced by deregulation of the Geminin/CDT1 balance is often incomplete and does not lead to real polyploidy. Ullah et al. (2008) find that Geminin levels remain un- 


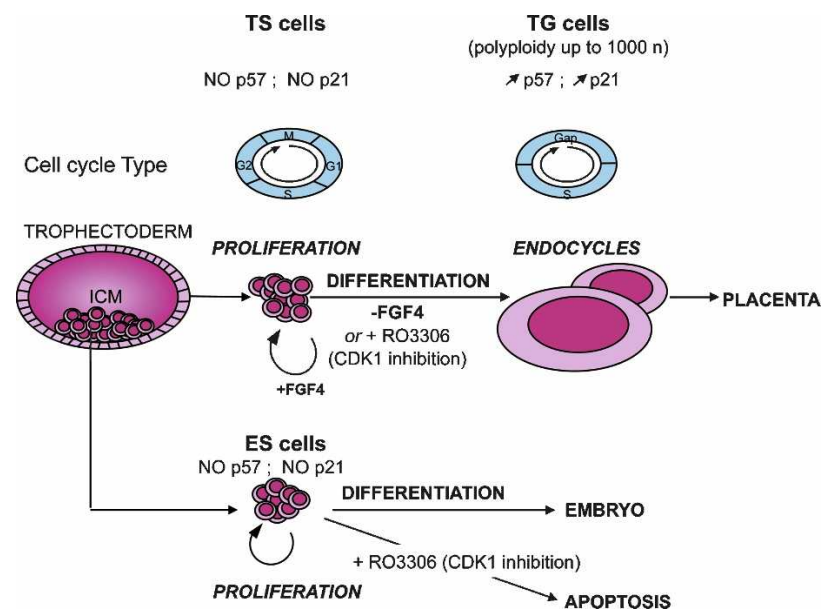

Figure 1. Coming and becoming of TG cells. Trophoblast Stem (TS) cells derive from the blastocyst trophectoderm. Upon FGF4 deprivation, they differentiate into TG cells, which participate in the formation of the placenta. TS cells, like embryonic stem (ES) cells, proliferate following the mitotic cell cycle that consists of sequences of DNA replication (S phase) and cell division (M phase), separated by two Gap phases (G1 and G2). When TS cells begin to differentiate, they stop proliferating and start endoreduplication, in which mitosis is aborted and S phases are separated by a unique Gap phase ( $\mathrm{G}$ phase). This process is concomitant with the specific induction of p57 and p21, which are expressed in TG cells undergoing endoreduplication, but not in ES or TS cells. Ullah et al. (2008) show that differentiation of TS cells into TG cells can be induced by inhibiting CDK1 activity with RO3306. By contrast, RO3306 induces apoptosis in ES cells.

changed during TS differentiation. These observations are consistent with a recent study by Zielke et al. (2008) showing that Geminin is necessary during endocycles in Drosophila as it is during the normal mitotic cycle. Taken together, these results suggest that Geminin activity participates in preventing rereplication during $S$ phase, but not in the setting of endocycles. Thus, Geminin-induced rereplication is likely to be involved in genome instability and DNA amplification linked to cancer, rather than in physiological endoreplication.

\section{Endoreplication by deregulation of cell cycle oscillators}

By contrast to polyploidy due to DNA overreplication during $\mathrm{S}$ phase (i.e., deregulation of the balance CDT1/ Geminin), endocycles may also result from abortive division. Thus, hereafter, "rereplication" will be used to describe endoreplication resulting from DNA overreplication during S phase, and "endoreduplication" will describe overploidy resulting from abortive mitosis.

Endoreduplication may occur through deregulation of the two main cell cycle oscillators that are conserved from yeast to mammals: CDKs and the ubiquitin ligases APC/C (anaphase-promoting complex/cyclosome) and SCF (SKP1-CUL1-F-box protein). In human cells, each step of the cell cycle can be characterized by the activity of specific Cyclin/CDK complexes. The G1 complexes
Cyclin D/CDK4-6 and Cyclin E/CDK2 drive progression through G1 and entry into $S$ phase, whereas the mitotic Cyclins A and B activate CDK1 to drive entry and progression through mitosis. CDKs are modulated by two classes of cyclin kinase inhibitors (CKIs) related to the p16INK4 family, which inhibits CDK4-6 activity and the p21CIP/WAF1 family, including p21, p27, and p57 (Besson et al. 2008). By opposition to the CDKs, CKIs function as negative regulators of the cell cycle and have been implicated in differentiation processes, mainly by coupling cell cycle arrest with differentiation (for review, see Besson et al. 2008). Endoreduplication can be observed after selective inhibition of CDK1 by conditional gene ablation (Itzhaki et al. 1997), drugs (Damiens et al. 2001;Vassilev 2006; Hochegger et al. 2007), or downregulation of the expression of both Cyclin B1 and B2 by siRNA (Bellanger et al. 2007). In all these cases, the polyploid cells exhibit discrete values of DNA content due to successive complete rounds of DNA replication without cell division.

APC/C and SCF constitute the second main oscillating regulator of cell cycle progression (Vodermaier 2004; Thornton and Toczyski 2006). They concomitantly control the ubiquitin-dependent degradation of key cell cycle factors, including Cyclins and CKIs. It is thus conceivable that at least one of these regulators might be altered during physiological endoreduplication.

In this issue of Genes \& Development, Ullah et al. (2008) analyze the expression of several key regulators of the mitotic cell cycle and provide strong evidence for a pivotal role of the CKIs p57 and p21 in CDK1 inhibition and the induction of endoreduplication of TG cells. Indeed, they report that induction of p57 and p21 is sufficient to promote endoreduplication and the atypical and specific survival of polyploid trophoblast cells.

\section{Endoreduplication and the CDK1 pathway}

To elucidate the involvement of CDK1 in TG cell endoreduplication, Ullah et al. (2008) used the specific chemical inhibitor of CDK1 RO3306 and took advantage of the high specificity of this inhibitor, which, unlike many other CDK inhibitors, does not interfere with CDK2 activity (Vassilev et al. 2006). Remarkably, they find that inhibition of CDK1 in TS cells induces their differentiation into TG cells and recapitulates the main events following FGF4 deprivation: endoreduplication and specific expression of differentiation markers. These findings provide strong evidence for the involvement of CDK1 inhibition in the natural endoreduplication of TS cells undergoing differentiation into TG cells. Then, Ullah et al. (2008) show that DNA replication itself during the endocycle is driven by CDK2, bound to Cyclin $\mathrm{E}$ or Cyclin A. Crucially, the investigators observe that physiological endoreduplication of TS cells elicited by FGF4 deprivation is correlated with rapid induction of both p57 and p21, the natural inhibitors of CDK1, but not of $\mathrm{p} 27$, which is involved in G1/S transition. This induction occurs conjointly with the dramatic loss of CDK1 activity. These findings corroborate the work by 
Hattori et al. (2000) showing that p57 expression is specifically induced during rodent TG cells differentiation. Taken together, the data presented by DePamphilis's group (Ullah et al. 2008) demonstrate that CDK1 inhibition occurs physiologically to drive endoreduplication in mammals.

Remarkably, DePamphilis's group (Ullah et al. 2008) shows that Cyclin B1 level remains unchanged throughout the endocycles, suggesting that the rapid loss of CDK1 activity is due to only p57 and p21 up-regulation. At the same time, Cyclin E and A decrease significantly. However, since Ullah et al. (2008) used only asynchronous cells, which did not allow the investigators to do a single cell analysis, it cannot be excluded that Cyclin E and Cyclin A levels oscillate during the endocycles, as previously described by MacAuley et al. (1998) in rodent TG cells. Further investigations concerning the expression of all these proteins by FACS or by immunofluorescence should clarify these differences and precisely define the exact timing of action of each molecule involved in the regulation of endocycles.

\section{The involvement of $\mathrm{p} 57$ in endoreplication in TG cells}

Ullah et al. (2008) then went on to specify the role of p57 and p21 during endoreplication in TG cells. They observe that, after FGF4 withdrawal, $p 21^{-/-}$TS cells endoreduplicate, whereas $p 57^{-1-}$ TS cells cannot any longer. These findings highlight the central role of p57 in endocycles. The investigators then clearly demonstrated the existence of a close relationship between CDK1 activity and $\mathrm{p} 57$ by showing that endoreduplication in $p 57^{-/-} \mathrm{TS}$ cells can be fully rescued through inhibition of CDK1 with RO3306. Interestingly, $p 57^{-/-}$TS cells continue to proliferate after FGF4 deprivation and start to become multinucleated, due to a failure of cytokinesis, and not due to endoreduplication. Multinucleation in $p 57^{-/-}$TG cells might have the same consequence as endoreduplication, suggesting that gene dosage augmentation is one facet of the differentiation process. Remarkably, these findings recapitulate the placental phenotype observed in $p 57^{-1-}$ mice: a delay in differentiation of trophoblast cells, which continue to proliferate, leading to placentomegaly, a phenotype that does not seem to interfere drastically with the function of the placenta since $p 57^{-/-}$ embryos develop to birth (Yan et al. 1997; Takahashi et al. 2000, Kanayama et al. 2002).

Ullah et al. (2008) also show that $p 57^{-1-}$ TS cells differentiate after FGF4 deprivation, as shown by the expression of several differentiation markers, even though they do not endoreduplicate. Taken together, these data demonstrate that the mechanism of endoreduplication is not itself involved in the differentiation process and that TS cells are already programmed to differentiate.

An unexpected function of $\mathrm{p} 21$ in the suppression of apoptosis induced by endoreduplication in TG cells

Ullah et al. (2008) observe that differentiation of TS cells into TG cells is accompanied by rapid and dramatic in- duction of p21 even though it does not play a role in the induction of endoreduplication. FGF4 deprivation suppresses the ATR-CHK1 safeguard pathway in endoreduplicating TG cells, allowing their survival. Indeed, in other cell types, endoreduplication activates the ATR-CHK1 pathway, which triggers apoptosis. During TS differentiation, the investigators find a strong correlation between $\mathrm{p} 21$ and $\mathrm{p} 57$ expression and CHK1 disappearance, suggesting that these CKIs inhibit apoptosis in TG cells through suppression of ATR and CHK1 activity. In $p 57^{-/-}$TS cells, upon FGF4 deprivation, p21 is induced and CHK1 efficiently suppressed, thus indicating that the primary role of p21 up-regulation in TS cells following FGF4 deprivation might be to prevent apoptosis (see Fig. 2). These findings confirm a previous study showing that p21 can suppress expression of CHK1 in human colorectal cancer cells (Gottifredi et al. 2001). Ullah et al. (2008) also show that both p57 and p21 are able to suppress CHK1 expression since $p 21^{-/-}$TS cells were still able to endoreduplicate. This partial redundancy of p57 and p21 in endoreduplicating TG cells confirm the in vivo observations by Zhang et al. (1999), who showed that concomitant loss of p21 exacerbates the placental defects observed in $p 57^{-/-}$mice. However, the complete mechanism involved in this inhibition of apoptosis remains to be elucidated. Notably, it should be important to explain how p21 and p57 trigger the suppression of CHK1 expression.

\section{Cyclic endocycles}

An intriguing result that emerges from the work by U1lah et al. (2008) is the ability of CDK1 to replace CDK2, as $C D K 2^{-/-}$TG cells endoreduplicate. Based on this observation, the investigators hypothesized that CDK1 inhibition in these cells is not constant but oscillates during endocycles. Since p57 is a potent inhibitor of both

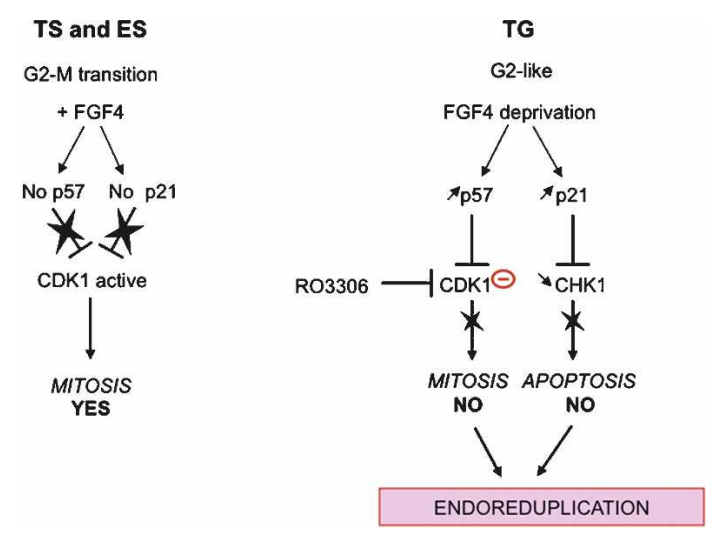

Figure 2. Molecular basis of endoreduplication in TG cells. Unlike ES and TS cells, TG cells that undergo endoreduplication express both $\mathrm{p} 21$ and $\mathrm{p} 57$. While $\mathrm{p} 57$ inhibits CDK1 function and, therefore, entry into mitosis, p21 suppresses CHK1 activity, thus avoiding apoptosis of TG cells. Endoreduplication in TG cells can be recapitulated in the presence of FGF4 by inhibiting CDK1 activity with RO3306. 
CDK1 and CDK2, CDK oscillation could be triggered by p57 oscillation. To assess this hypothesis, DePamphilis's group (Ullah et al. 2008) analyzed the expression of p57 throughout the endocycle and finds that it is restricted to cells in Gap phase and absent in cells in S phase. Thus, p57 expression in G2 would inhibit CDK1 activity and M-phase entry, whereas its absence at the G1/S transition would allow CDK2 to be activated and trigger DNA replication. These findings confirm those made by Hattori et al. (2000), who also described p57 oscillations during rodent TG cells endoreduplication. Although oscillation of $\mathrm{p} 57$ has been well established, p21 expression during endoreduplication of TG cells remains to be clarified.

Since p57 mRNA level does not change during endocycles in both human TG cells and rat choriocarcinoma (Rcho1) cells (Hattori et al. 2000; Ullah et al. 2008), p57 oscillations might be controlled post-transcriptionally by regulators, which may themselves oscillate. This model is supported by previous observations in other cells lines that showed that p57 phosphorylation by Cyclin E/CDK2 leads to p57 degradation through the E3-ubiquitin protein ligase $\mathrm{SCF}^{\text {Skp2 }}$ pathway (Kamura et al. 2003). It is thus not surprising that a nonphosphorytable mutant of p57 induces its own stabilization and inhibits endoreduplication in rodent TG cells (Hattori et al. 2000). Furthermore, it could be interesting to analyze p57 behavior in Cyclin $E 1^{-/-} / E 2^{-/-}$double knockout $(\mathrm{KO})$ embryos or in Cyclin $E 1^{-/-} / \mathrm{E}^{-/-} \mathrm{TS}$ cells undergoing differentiation, and to address whether the placental defects observed in this background result from deregulation of p57 expression.

\section{Endoreduplication is not the result of a universally conserved mechanism}

As stressed by Ullah et al. (2008), the mechanism that triggers endoreduplication in human TS cells is not evolutionary conserved. Indeed, $p 57$ and p21 do not exist in Drosophila melanogaster; nevertheless, several of its tissues initiate endocycles during embryogenesis and in adult life. However, as in humans, the final target of endoreduplication in Drosophila is again CDK1. Similarly, it is interesting to note that in mice, KO experiments showed that CDK1 is the only essential cell cycle CDK (Santamaria et al. 2007). In flies, transition into endocycles is driven by down-regulation of String/Cdc25 transcription, resulting in the inhibition of CDK1 activity. At the same time, premature expression of Fzr induces the degradation of mitotic Cyclins and Geminin (Schaeffer et al. 2004; Narbonne-Reveau et al. 2008; Zielke et al. 2008). Thus, entry into mitosis is inhibited while, simultaneously, pre-RC formation is allowed.

Ullah et al. (2008) clearly show that endoreduplication in human TS cells undergoing differentiation into TG cells is not initiated by a similar pathway, but by the up-regulation of $\mathrm{p} 57$, which inhibits CDK1 activity regardless the expression of Cyclin A and B. Then, the subsequent endocycles are driven by the same oscillators: Cyclin E/CDK2, APC/CDH1 and p57. Intriguingly, in mammals, Cyclin E and $\mathrm{CDH} 1$ are necessary for endocycles, but not for mitotic cell cycles, as revealed by KO mutants (Geng et al. 2003; Parisi et al. 2003, GarciaHiguera et al. 2008; Li et al. 2008). Which of these three is the master of endocycles remains elusive. Further analysis concerning TS cells derived from these KO blastocysts should establish the hierarchy between these regulators.

\section{Differentiated cell cycles}

In this issue of Genes \& Development, Ullah et al. (2008) decipher the central role played by p57 and p 21 in the differentiation of TS into TG cells, and highlight once more the crucial function of CDK1 in driving the cell cycle. One important molecular aspect of TG differentiation is the concomitant induction of atypical endocycles and inhibition of apoptosis, which is normally induced after endoreduplication. Together, they ensure the generation and survival of endoreduplicated TG cells. Although p57 expression can be sufficient to trigger endoreduplication in TS cells, it is remarkable that it does not induce endoreduplication in other cell lines. Indeed, p57 has previously been implicated in the differentiation of various cell lines, which do not undergo endoreduplication, such as pancreatic cells (Georgia et al. 2006), keratinocytes (Gosselet et al. 2007), oligodendrocytes (Dugas et al. 2007), or intestinal epithelial cells (Riccio et al. 2008). These findings suggest that p57 up-regulation induces endoreduplication only in cells already programmed to endoreduplicate, indicating that a least another actor, which remains to be identified, is involved in the specific response to CDK1 inhibition. Therefore, it could be attractive to compare the mechanisms involved in TG endoreduplication with those triggering megakaryocyte endoreduplication.

The study by Ullah et al. (2008) illustrates how the intracellular context drives different answers to a similar stimulus, a concept already suggested by the analysis of the phenotypes induced by invalidation of the regulators of the cell cycle (for review, see Malumbres and Barbacid 2005). Surprisingly, only restricted cell types are affected. It is thus tempting to speculate about the existence of a combinatory system of cell cycle regulations, which may be adapted to the differentiation behavior of the cell. Different combinations of cell cycle regulators may, ultimately, trigger tissue specificity in the presence of an identical extracellular stimulus. In a similar fashion, deregulation of specific cell cycle regulators may affect specific tissues without affecting the basic cell cycle properties. Thus, the cell cycle appears highly dependent on the cell type and cell cycle regulations cannot be defined by a universal and fixed network.

Similarly, if efforts during the past decades have failed to find a universal treatment for cell cycle diseases, such as cancer, they have highlighted the differences in each cancer cell type, suggesting that the elucidation of the specific combination of cell cycle factors adapted to each situation may help to increase target specificity. 


\section{Acknowledgments}

We thank Malik Lutzmann and Philippe Coulombe for critical reading of the manuscript.

\section{References}

Arias, E.E. and Walter, J.C. 2005. Replication-dependent destruction of Cdt1 limits DNA replication to a single round per cell cycle in Xenopus egg extracts. Genes \& Dev. 19: 114-126.

Bellanger, S., de Gramont, A., and Sobczak-Thepot, J. 2007. Cyclin B2 suppresses mitotic failure and DNA re-replication in human somatic cells knocked down for both cyclins B1 and B2. Oncogene 26: 7175-7184.

Besson, A., Dowdy, S.F., and Roberts, J.M. 2008. CDK inhibitors: Cell cycle regulators and beyond. Dev. Cell 14: 159169.

Calvi, B.R. 2006. Developmental gene amplification. In DNA replication and human disease (ed. M.L. DePamphilis), pp. 233-256. Cold Spring Harbor Laboratory Press, Cold Spring Harbor, NY.

Damiens, E., Baratte, B., Marie, D., Eisenbrand, G., and Meijer, L. 2001. Anti-mitotic properties of indirubin-3'-monoxime, a CDK/GSK-3 inhibitor: Induction of endoreplication following prophase arrest. Oncogene 20: 3786-3797.

DePamphilis, M.L., Blow, J.J., Ghosh, S., Saha, T., Noguchi, K., and Vassilev, A. 2006. Regulating the licensing of DNA replication origins in metazoa. Curr. Opin. Cell Biol. 18: 231239

Dugas, J.C., Ibrahim, A., and Barres, B.A. 2007. A crucial role for p57(Kip2) in the intracellular timer that controls oligodendrocyte differentiation. J. Neurosci. 27: 6185-6196.

Edgar, B.A. and Orr-Weaver, T.L. 2001. Endoreplication cell cycles: More for less. Cell 105: 297-306.

Garcia-Higuera, I., Manchado, E., Dubus, P., Canamero, M., Mendez, J., Moreno, S., and Malumbres, M. 2008. Genomic stability and tumour suppression by the APC/C cofactor Cdh1. Nat. Cell Biol. 10: 802-811.

Geng, Y., Yu, Q., Sicinska, E., Das, M., Schneider, J.E., Bhattacharya, S., Rideout, W.M., Bronson, R.T., Gardner, H., and Sicinski, P. 2003. Cyclin E ablation in the mouse. Cell 114: 431-443.

Georgia, S., Soliz, R., Li, M., Zhang, P., and Bhushan, A. 2006. p57 and Hes1 coordinate cell cycle exit with self-renewal of pancreatic progenitors. Dev. Biol. 298: 22-31.

Goga, A., Yang, D., Tward, A.D., Morgan, D.O., and Bishop, J.M. 2007. Inhibition of CDK1 as a potential therapy for tumors over-expressing MYC. Nat. Med. 13: 820-827.

Gonzalez, M.A., Tachibana, K.E., Adams, D.J., van der Weyden, L., Hemberger, M., Coleman, N., Bradley, A., and Laskey, R.A. 2006. Geminin is essential to prevent endoreduplication and to form pluripotent cells during mammalian development. Genes \& Dev. 20: 1880-1884.

Gosselet, F.P., Magnaldo, T., Culerrier, R.M., Sarasin, A., and Ehrhart, J.C. 2007. BMP2 and BMP6 control p57(Kip2) expression and cell growth arrest/terminal differentiation in normal primary human epidermal keratinocytes. Cell. Signal. 19: 731-739.

Gottifredi, V., Karni-Schmidt, O., Shieh, S.S., and Prives, C. 2001. p53 down-regulates CHK1 through p21 and the retinoblastoma protein. Mol. Cell. Biol. 21: 1066-1076.

Hara, K., Nakayama, K.I., and Nakayama, K. 2006. Geminin is essential for the development of preimplantation mouse embryos. Genes Cells 11: 1281-1293.
Hattori, N., Davies, T.C., Anson-Cartwright, L., and Cross, J.C. 2000. Periodic expression of the cyclin-dependent kinase inhibitor p57(Kip2) in trophoblast giant cells defines a G2-like gap phase of the endocycle. Mol. Biol. Cell 11: 1037-1045.

Hochegger, H., Dejsuphong, D., Sonoda, E., Saberi, A., Rajendra, E., Kirk, J., Hunt, T., and Takeda, S. 2007. An essential role for Cdk1 in S phase control is revealed via chemical genetics in vertebrate cells. I. Cell Biol. 178: 257-268.

Itzhaki, J.E., Gilbert, C.S., and Porter, A.C. 1997. Construction by gene targeting in human cells of a "conditional" CDC2 mutant that rereplicates its DNA. Nat. Genet. 15: 258-265.

Kamura, T., Hara, T., Kotoshiba, S., Yada, M., Ishida, N., Imaki, H., Hatakeyama, S., Nakayama, K., and Nakayama, K.I. 2003. Degradation of p57Kip2 mediated by SCFSkp2-dependent ubiquitylation. Proc. Natl. Acad. Sci. 100: 10231-10236.

Kanayama, N., Takahashi, K., Matsuura, T., Sugimura, M., Kobayashi, T., Moniwa, N., Tomita, M., and Nakayama, K. 2002. Deficiency in p57Kip2 expression induces preeclampsia-like symptoms in mice. Mol. Hum. Reprod. 8: 1129-1135.

Li, A. and Blow, J.J. 2005. Cdt1 downregulation by proteolysis and geminin inhibition prevents DNA re-replication in Xenopus. EMBO I. 24: 395-404.

Li, M., Shin, Y.H., Hou, L., Huang, X., Wei, Z., Klann, E., and Zhang, P. 2008. The adaptor protein of the anaphase promoting complex Cdh1 is essential in maintaining replicative lifespan and in learning and memory. Nat. Cell Biol. 10: 1083-1089.

MacAuley, A., Cross, J.C., and Werb, Z. 1998. Reprogramming the cell cycle for endoreduplication in rodent trophoblast cells. Mol. Biol. Cell 9: 795-807.

Maiorano, D., Krasinska, L., Lutzmann, M., and Mechali, M. 2005. Recombinant Cdt1 induces rereplication of G2 nuclei in Xenopus egg extracts. Curr. Biol. 15: 146-153.

Malumbres, M. and Barbacid, M. 2005. Mammalian cyclin-dependent kinases. Trends Biochem. Sci. 30: 630-641.

Mihaylov, I.S., Kondo, T., Jones, L., Ryzhikov, S., Tanaka, J., Zheng, J., Higa, L.A., Minamino, N., Cooley, L., and Zhang, H. 2002. Control of DNA replication and chromosome ploidy by geminin and cyclin A. Mol. Cell. Biol. 22: 18681880.

Narbonne-Reveau, K., Senger, S., Pal, M., Herr, A., Richardson, H.E., Asano, M., Deak, P., and Lilly, M.A. 2008. APC/CFzr/ Cdh1 promotes cell cycle progression during the Drosophila endocycle. Development 135: 1451-1461.

Parisi, T., Beck, A.R., Rougier, N., McNeil, T., Lucian, L., Werb, Z., and Amati, B. 2003. Cyclins E1 and E2 are required for endoreplication in placental trophoblast giant cells. EMBO J. 22: 4794-4803.

Riccio, O., van Gijn, M.E., Bezdek, A.C., Pellegrinet, L., van Es, J.H., Zimber-Strobl, U., Strobl, L.J., Honjo, T., Clevers, H., and Radtke, F. 2008. Loss of intestinal crypt progenitor cells owing to inactivation of both Notch1 and Notch2 is accompanied by derepression of CDK inhibitors p27Kip1 and p57Kip2. EMBO Rep. 9: 377-383.

Santamaria, D., Barriere, C., Cerqueira, A., Hunt, S., Tardy, C., Newton, K., Caceres, J.F., Dubus, P., Malumbres, M., and Barbacid, M. 2007. Cdk1 is sufficient to drive the mammalian cell cycle. Nature 448: 811-815.

Schaeffer, V., Althauser, C., Shcherbata, H.R., Deng, W.M., and Ruohola-Baker, H. 2004. Notch-dependent Fizzy-related/ $\mathrm{Hec} 1 / \mathrm{Cdh} 1$ expression is required for the mitotic-to-endocycle transition in Drosophila follicle cells. Curr. Biol. 14: 630-636.

Simmons, D.G. and Cross, J.C. 2005. Determinants of trophoblast lineage and cell subtype specification in the mouse placenta. Dev. Biol. 284: 12-24. 
Takahashi, K., Kobayashi, T., and Kanayama, N. 2000. p57(Kip2) regulates the proper development of labyrinthine and spongiotrophoblasts. Mol. Hum. Reprod. 6: 1019-1025.

Thomer, M., May, N.R., Aggarwal, B.D., Kwok, G., and Calvi, B.R. 2004. Drosophila double-parked is sufficient to induce re-replication during development and is regulated by cyclin E/CDK2. Development 131: 4807-4818.

Thornton, B.R. and Toczyski, D.P. 2006. Precise destruction: An emerging picture of the APC. Genes \& Dev. 20: 30693078.

Ullah, Z., Kohn, M.J., Yagi, R., Vassilev, L.T., and DePamphilis, M.L. 2008. Differentiation of trophoblast stem cells into giant cells is triggered by p57/Kip2 inhibition of CDK1 activity. Genes \& Dev. (this issue). doi: 10.1101/gad.1718108.

Vassilev, L.T. 2006. Cell cycle synchronization at the G2/M phase border by reversible inhibition of CDK1. Cell Cycle 5: 2555-2556.

Vassilev, L.T., Tovar, C., Chen, S., Knezevic, D., Zhao, X., Sun, H., Heimbrook, D.C., and Chen, L. 2006. Selective smallmolecule inhibitor reveals critical mitotic functions of human CDK1. Proc. Nat1. Acad. Sci. 103: 10660-10665.

Vodermaier, H.C. 2004. APC/C and SCF: Controlling each other and the cell cycle. Curr. Biol. 14: R787-R796. doi: 10.1016/ j.cub.2004.09.020.

Yan, Y., Frisen, J., Lee, M.H., Massague, J., and Barbacid, M. 1997. Ablation of the CDK inhibitor p57Kip2 results in increased apoptosis and delayed differentiation during mouse development. Genes \& Dev. 11: 973-983.

Zhang, P., Wong, C., Liu, D., Finegold, M., Harper, J.W., and Elledge, S.J. 1999. p21(CIP1) and p57(KIP2) control muscle differentiation at the myogenin step. Genes \& Dev. 13: 213224.

Zhu, W., Chen, Y., and Dutta, A. 2004. Rereplication by depletion of geminin is seen regardless of p53 status and activates a G2/M checkpoint. Mol. Cell. Biol. 24: 7140-7150.

Zielke, N., Querings, S., Rottig, C., Lehner, C., and Sprenger, F. 2008. The anaphase-promoting complex/cyclosome (APC/C) is required for rereplication control in endoreplication cycles. Genes \& Dev. 22: 1690-1703. 


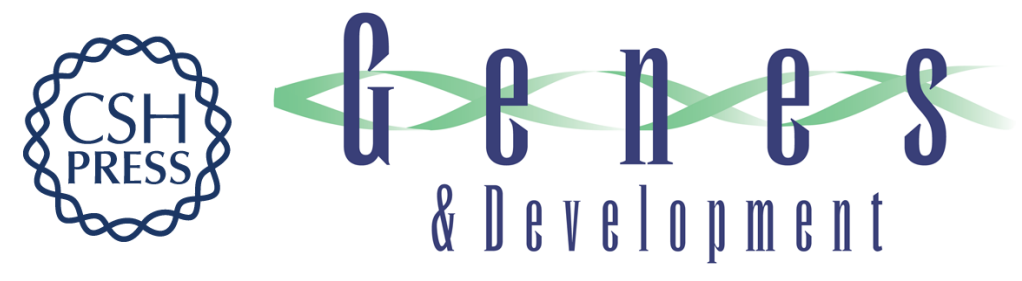

\section{New cell or new cycle?}

Olivier Ganier and Marcel Mechali

Genes Dev. 2008, 22:

Access the most recent version at doi:10.1101/gad.1740808

\section{Related Content Differentiation of trophoblast stem cells into giant cells is triggered by p57/Kip2 inhibition of CDK1 activity \\ Zakir Ullah, Matthew J. Kohn, Rieko Yagi, et al. \\ Genes Dev. November , 2008 22: 3024-3036}

References This article cites 43 articles, 20 of which can be accessed free at:

http://genesdev.cshlp.org/content/22/21/2908.full.html\#ref-list-1

Articles cited in:

http://genesdev.cshlp.org/content/22/21/2908.full.html\#related-urls

\section{License}

Email Alerting Receive free email alerts when new articles cite this article - sign up in the box at the top Service right corner of the article or click here.

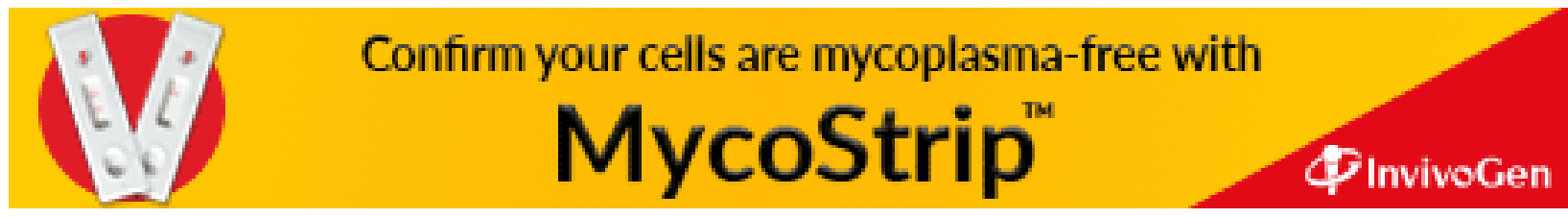

